\title{
Tuberous sclerosis with attention deficit hyperactivity disorder- A case report
}

\author{
Ichpreet Singh ${ }^{1}$, Prajakta Patkar ${ }^{2}$, Suprakash Chaudhury ${ }^{3, *}$, D. Saldanha ${ }^{4}$, Chetan Diwan ${ }^{5}$ \\ ${ }^{1,2}$ Resident ${ }^{3}$ Professor, ${ }^{4}$ Professor and HOD, ${ }^{5}$ Assistant Professor, ${ }^{1-4}$ Dept of Psychiatry, ${ }^{5}$ Dept. of Social Work, ${ }^{1-4}$ Dr. D Y Patil Medical \\ College, Hospital and Research Center, Pimpri, Pune, Maharashtra, ${ }^{5}$ Karve Institute of Social Service, Pune, Maharashtra, India
}

\section{*Corresponding Author:}

Email: suprakashch@gmail.com

\begin{abstract}
A patient with tuberous sclerosis and attention deficit hyperactivity disorders is reported. He presented with generalized tonic-clonic seizures, skin lesions, restlessness, irritability, hyperactivity and impulsivity since 4 years. He had also developed hyperactivity and impulsivity for the past 2 years. Examination showed Shagreen patches and multiple hypomelanotic macules. MRI brain revealed multiple cortical tubers and sub-ependymal nodules. The condition is briefly discussed.
\end{abstract}

Keywords: Tuberous sclerosis, Attention deficit hyperactivity disorder, Tuberous sclerosis associated neuropsychiatric disorders.

\section{Introduction}

Tuberous sclerosis (TS) is a multi-systemic autosomal dominant genetic disease. In $85 \%$ of cases a mutation is present in 1 of the 2 tumor suppressor genes, TSC1 on chromosome $9 \mathrm{q} 34$ or TSC2 on chromosome 16p13. TS has a prevalence of 1 of 6000 newborn and worldwide an estimated 1.5 million suffer from it. ${ }^{1}$ TS is characterized by multiple benign hamartomatous lesions present in the skin, brain, kidneys, eyes, or heart. $^{2}$ Attention-deficit hyperactivity disorder (ADHD) is a common neurobehavioral disorder with onset in early childhood, caused by the presence of many genetic and environmental risk factors. The main symptom which is seen in ADHD is inappropriate and impaired motor activity respective to their age along with over activity, inattention and impulsivity. ADHD is often diagnosed in individuals with neurogenetic disorders such as neurofibromatosis, Prader-Willi syndrome, fragile X syndrome Turner syndrome, William's syndrome, velocardiofacial syndrome, and tuberous sclerosis. A patient of TS with intellectual disability and ADHD is reported here to highlight the Tuberous Sclerosis Associated Neuropsychiatric Disorders (TAND). ${ }^{1}$

\section{Case History}

A 7 year-old-male child was brought to the hospital by his parents with complaints of skin lesions, restlessness, irritability, hyperactivity and impulsivity since 2 years. The patient was apparently alright 4 years ago when he started having generalized tonic-clonic type seizures associated with frothing from mouth and urinary incontinence which lasted around 1-2 minutes associated with post episodic confusion. As these used to occur every 10-15 days, the parents thus visited a doctor and was started on Tab phenytoin. Subsequently the frequency of episodes reduced to 1-2 in a year. Around 2 years back, he started developing small lesions on his face and trunk. The lesions were small pinpoint in size, painless and did not itch. Earlier they were only two- three on the cheeks and two-three on the trunk, but later it gradually progressed to multiple in the last few months. The next complaint by the mother was poor understanding or intelligence. She said that he found it difficult to follow simple instructions. He would get confused if more than one thing was instructed to him at one time. He had to be assisted to do day to day activities like getting dressed and even having a bath or using the washroom. She said that the children of his age seemed to be smarter than him and he would lag behind. The patient couldn't get along with the children of his age group as he wouldn't understand the play and hence would prefer playing with a much younger bunch of kids. The child's mother also complained of hyperactivity along with impulsivity in the last 2 years. He seemed restless the whole day and if doing any task couldn't be at it for more than 4-5 minutes. He would be easily distractible from whatever he is doing by minor distractions and would leave the task unfinished and switch to something else. He would also not come back to the unfinished task again. Mother reported he had become very fidgety and restless and couldn't sit in one place for long. He would often be irritable without reason. The child was observed to not be able to wait for his turn and often snatch things from people's hands. He would many a times speak at inappropriate times and interrupt people. His past history and family history didn't reveal anything significant.

The child was born out of a non-consanguineous marriage at 9 months of gestation. He was a full tem normal vaginal delivery in the hospital. No antepartum or postpartum complications. Motor development was appropriate to age. Speech development was delayed. He started talking at 4 years of age, currently had a vocabulary of 10-15 words.

On examination, he was conscious, non-cooperative, restless, irritable and hyperactive. He was unable to sit at one place for more than 5-10secs. His vitals were stable. BP was 98/70mm hg, Pulse 110 beats/min and RR-29/min. The child had hyper pigmented patches on the face on cheeks. There were multiple lesions on the lower back looking like thick leathery skin that are dimpled like an orange peel. Multiple hypo-pigmented macules were present over the buttocks and trunk (Fig. 1 and 2) All the lesions were non pruritic and no signs of inflammation were seen. There were no signs of icterus, cyanosis, clubbing, lymphadenopathy. 
On systemic examination, his cardiovascular, respiratory and per abdomen examination were normal. On neurological examination, he was conscious, cooperative, no gross motor or sensory abnormalities. On mental status examination, he was shy, calm and respectful towards the examiner. He was relevant and coherent with no features of psychosis.

Routine hematological parameters, chest X-ray, USG (abdomen and pelvis), 2D-echo were all within normal limits. MRI brain revealed altered signal intensities in the cortical and subcortical grey matter of bilateral frontal, parietal, occipital and right temporal lobes and parafalcine region appearing hyperintense on $\mathrm{T} 2 \mathrm{~W}$ and FLAIR sequences and isointense on T1W sequences. All these findings were indicative of cortical tubers. Few subependymal nodules were observed appearing hypointense on $\mathrm{T} 2 \mathrm{~W}$ and isointense on $\mathrm{T} 1 \mathrm{~W}$. Cortical thickening was noted in bilateral occipital lobes with effacement of gyri. All these findings were suggestive of tuberous sclerosis. Due to his hyperactivity, impulsivity and speech delay, a formal IQ assessment could not be performed. On Vineland Social Maturity Scale his mental age was 3 years.

The child was then started on Tab. Methylphenidate, at a dose of $10 \mathrm{mg}$ per day. He showed significant improvement in his hyperactivity within 20 days. Parents were advised about the importance of medications and follow up. They were also counseled about his intellectual disability and its prognosis. They were also recommended the need for a special institute for training him and at least making him self-sufficient for his day to day activities.

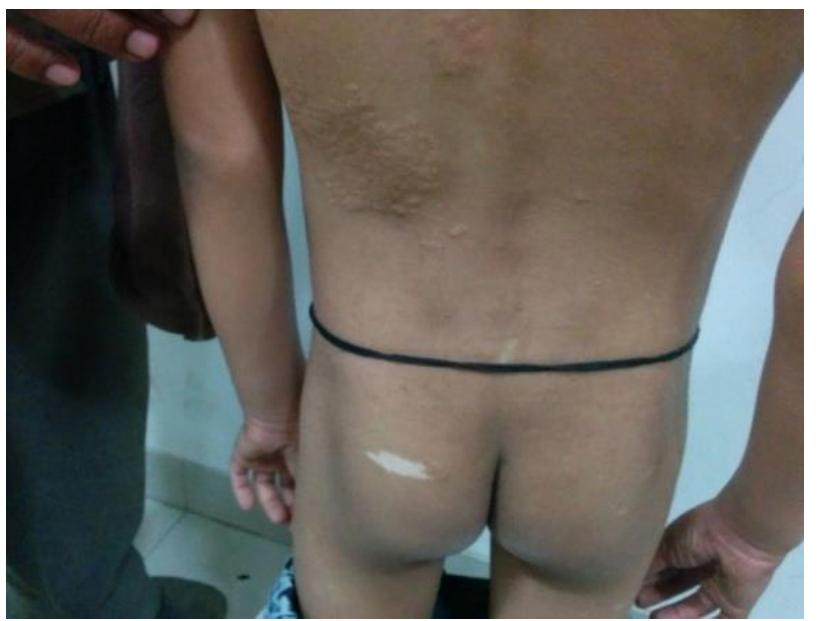

Fig. 1: Shagreen patches and multiple hypomelanotic macules

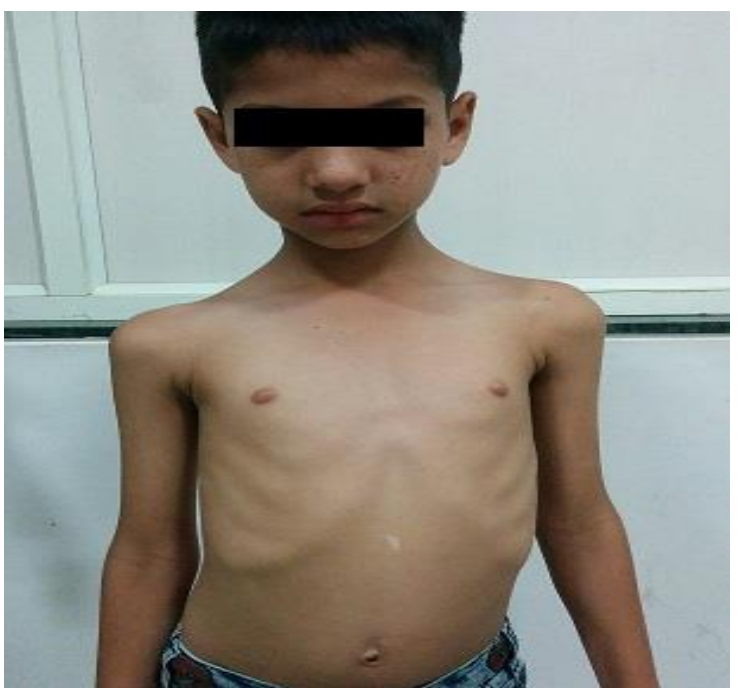

Fig. 2: Facial angiofibroma and hypomelanotic macule

\section{Discussion}

TS was named by Desire-Magloire Bourneville who also gave the first detailed description of its cerebral pathology. ${ }^{3}$ The classic diagnostic triad for tuberous sclerosis complex consisting of mental retardation, epilepsy, and adenoma sebaceum was first described by Vogt in $1908 .{ }^{4} \mathrm{TS}$ is a genetically determined but variably expressed disorder. In familial cases it is an autosomal dominant disorder while in others it is a sporadic mutation. It is a multisystem disorder that produces well circumscribed, benign, and noninvasive lesions in any human organ most often the skin, brain, retina, heart, kidney, and lung. Majority of the patients are identified when they present with CNS manifestations. ${ }^{1}$ Brain lesions are present in almost all cases and hence CNS manifestations are the hallmark of TSC. The brain lesions of TS can be distinguished by neuroimaging studies and include subependymal nodules in $80 \%$ individuals, cortical dysplasias in $90 \%$ and subependymal giant cell astrocytomas in 5-15\%.5 Of all of the possible manifestations of this complex disorder, however, the cognitive and behavioral problems represent the area of greatest concern to parents and caregivers.

Problems faced in day-to-day life by parents, caregivers, teachers, or others are described as behavioral difficulties. Common behavioral difficulties in TS include anxiety, depression, aggression, temper tantrums, difficulty concentrating, hyperactivity, impulsivity, speech and language delays, poor eye contact, and difficulties in relationships with peers, repetitive behaviors, self-injurious behaviors, and eating or sleep difficulties. ${ }^{6-9}$ Even though approximately $90 \%$ of subjects with TS have one or more TAND concerns only $20 \%$ receive evaluation and intervention for the same. ${ }^{1}$ Psychiatric disorders commonly seen in subjects with TS include autism spectrum disorders (25\%-50\%), ADHD (30\%-50\%), anxiety and depressive disorders $(30 \%-60 \%) .6,7,10$ 
Intellectual disability is observed in $50 \%$ of individuals with TS, out of which $30 \%$ are in profound level. ${ }^{11,12}$ According to a study done in Boston, about $66 \%$ patients fell into at least one psychiatric symptom group, with $32 \%$ patients having been treated with a psychiatric medication. $30 \%$ patients had a history of ADHD-type behavior. ${ }^{13}$

Specific learning disability is seen in $30 \%$ of school aged TS children having normal IQ. ${ }^{11}$ Neuropsychological evaluations in TS commonly show specific deficits in working memory, cognitive flexibility, or dual tasking. ${ }^{4-15}$ This 7-year-old child brought in by his parents came with seizure disorder along with behavioral abnormalities. Although his seizure disorder was under control, the new onset issues with inattention and hyperactivity and the dermatological changes were of concern to the parents. His intellectual disability and speech delay made it all the more difficult to be managed at home. Managing him behaviorally was as essential as the parents counseling about the nature and prognosis of his multiple problems. The most important part in his management was the long term rehabilitation.

\section{Conclusion}

Along with the medical management, an early diagnosis and management of psychiatric and behavioral issues of patients suffering from tuberous sclerosis is warranted. Identifying and acknowledging behavioral problems should lead to determining whether any specific psychiatric disorder might be present to investigate the possible causes of the behavioral difficulty and to implement an appropriate management strategy. Psycho education of the parents and caregivers about the disease and its multisystem implications is equally necessary.

\section{Conflict of Interest: None.}

\section{References}

1. deVries PJ, Whittemore VH, Leclezio L, Byars AW, Dunn D, Ess KC, et al. Tuberous sclerosis associated neuropsychiatric disorders (TAND) and the TAND checklist. Pediatr Neurol 2015;52(1):25-35.

2. Baskin HJ. The pathogenesis and imaging of the tuberous sclerosis complex. Pediatr Radiol 2008;38:936-952.

3. Bourneville DM. Sclerose tubereuse des circonvolutions cerebrales: idiotie et epilepaie hemiplegique. Arch Neural (Paris) 1880:1:81-91.

4. Vogt H. Zur Pathologie und pathologischen Anatomie der verschiedenen Idiotieform. Hfonatsschr Psyzhratr Neural 1908:24:106650.
5. Northrup H, Krueger DA. International Tuberous Sclerosis Complex Consensus Group. Tuberous sclerosis complex diagnostic criteria update: recommendations of the 2012 International Tuberous Sclerosis Complex Consensus Conference. Pediatr Neurol 2013;2013;49:243-254.

6. de Vries, PJ. Neurodevelopmental, psychiatric and cognitive aspects of tuberous sclerosis complex. In: Kwiatkowski, DJ.; Whittemore, VH.; Thiele, EA., editors. Tuberous Sclerosis Complex: Genes, Clinical Features and Therapeutics. Weinheim, Germany: Wiley-Blackwell; 2010a. p. 229-267.

7. Prather P, de Vries PJ. Behavioral and cognitive aspects of tuberous sclerosis complex. J Child Neurol 2004;19:666-674.

8. de Vries PJ, Hunt A, Bolton PF. The psychopathologies of children and adolescents with tuberous sclerosis complex (TSC): a postal survey of UK families. Eur Child Adolesc Psychiatry 2007;16:16-24.

9. Eden KE, de Vries PJ, Moss J, Richards C, Oliver C. Selfinjury and aggression in tuberous sclerosis complex: crosssyndrome comparison and associated risk markers. J Neurodev Disord 2014; 6:10.

10. de Vries PJ. Targeted treatments for cognitive and neurodevelopmental disorders in tuberous sclerosis complex. Neurotherapeutics 2010b;7:275-282.

11. Joinson C, O'Callaghan FJ, Osborne JP, Martyn C, Harris T, Bolton PF. Learning disability and epilepsy in an epidemiological sample of individuals with tuberous sclerosis complex. Psychol Med 2003; 33:335-344.

12. de Vries PJ, Prather PA. The tuberous sclerosis complex. $N$ Engl J Med 2007;356:392.

13. Muzykewicz, David A. Newberry P, Danforth N, Halpern EF, Thiele EA. Psychiatric co-morbid conditions in a clinic population of 241 patients with tuberous sclerosis complex. Epilepsy Behav 2007;11:506-513.

14. Ridler K, Suckling J, Higgins NJ. Neuroanatomical correlates of memory deficits in tuberous sclerosis complex. Cereb Cortex 2007; 17:261-271.

15. de Vries PJ, Gardiner J, Bolton PF. Neuropsychological attention deficits in tuberous sclerosis complex (TSC). Am J Med Genet A 2009;149A:387-395. 